Trauma Berufskrankh 2009 · 11 [Suppl 2] 146-153

DOI 10.1007/s10039-008-1467-9

Online publiziert: 18. Februar 2009

(c) Springer Medizin Verlag 2009

S. Rein · R. Grass · H. Zwipp

Klinik und Poliklinik für Unfall- und Wiederherstellungschirurgie, Universitätsklinikum Carl Gustav Carus

\title{
Suprakondyläre Femurfrakturen
}

\section{Epidemiologie und Frakturklassifikation}

\section{Epidemiologie}

\section{Begriffsdefinition}

In der Literatur existieren unterschiedliche Angaben zur Definition der suprakondylären Femurfrakturen:

- Neer et al. [22] legten die suprakondyläre Region als die distalen 3 Inches des Femurs fest, was 7,62 cm entspricht.

- Seinsheimer [28] und Culp et al. [8] spezifizierten die suprakondyläre Region des Femurs mit $9 \mathrm{~cm}$ proximal der Kniegelenklinie.

- Sowohl Schatzker u. Lambert [27] als auch Sisto et al. [30] schlossen alle Frakturen bis $15 \mathrm{~cm}$ proximal der Kniegelenklinie in ihre Studien der suprakondylären Femurfrakturen ein.

Im Allgemeinen werden alle Frakturen bis $15 \mathrm{~cm}$ oberhalb der Kniegelenklinie als suprakondyläre Femurfrakturen bezeichnet, im Falle von Stielkomponenten der Prothesen werden alle Frakturen bis $5 \mathrm{~cm}$ des proximalen Implantatendes berücksichtigt [32].

\section{Häufigkeit}

Über die Häufigkeit distaler Femurfrakturen existieren verschiedene Angaben, erhoben in größeren Patientenkollektiven. Bezogen auf die Gesamtzahl der Frakturen am Femur hatten die Brüche im distalen Drittel bei Kolmert u. Wulff [16] einen Anteil von 4\%, bei Regazzoni et al. [23] von 7\%, bei Ecke et al. [10] von $18 \%$ und bei Arneson et al. [4] von bis zu 31\%.

Im Krankengut der Polytraumatisierten, bei welchen der Bewegungsapparat mit $72-100 \%$ die am häufigsten betroffene Region darstellt, ist der Oberschenkel in $27-30 \%$ beteiligt [21].

Die Prävalenz von distalen Femurfrakturen wird in der Literatur mit $0,4 \%$ bei einer Inzidenz von 4,5/10 Einwohner/ Jahr von allen Frakturen des erwachsenen Menschen angegeben, wobei Männer doppelt so häufig betroffen sind als Frauen [7].

Das Durchschnittsalter von Patienten mit distalen Femurfrakturen beträgt 61 Jahre, wobei die Prävalenz der distalen Femurfraktur mit 62,5\% der über 50-Jährigen, $50 \%$ der über 65-Jährigen und 41,7\% der über 75 -Jährigen angegeben wird [7].

\section{Ätiopathogenese}

\section{Ursachen}

Als Prädisposition für eine suprakondyläre Fraktur, die zu den osteoporotischen Brüchen zu rechnen ist [7], wird die Implantation einer Knieendoprothese angesehen [9]. Zu den Risikofaktoren für eine suprakondyläre Femurfraktur zählen Erkrankungen, die zu Veränderungen der Knochenstruktur führen, wie $[12,13]$ :

- Osteoporose,

- Osteomalazie,

- Morbus Paget,

- rheumatoide Arthritis,

- Osteopenie, oft kortikoidinduziert,
- Osteolysen und

- Stressfrakturen.

Neurologische Erkrankungen, welche zu gehäuften Stürzen führen, beispielsweise Morbus Parkinson oder epileptische Anfälle, können ebenfalls Ursachen suprakondylärer Frakturen sein [8]. Eine Sonderform stellt die pathologische Fraktur bei Malignomen dar, für welche beispielsweise Metastasen von Tumoren der Mamma oder der Niere verantwortlich sein können [18]. Weiterhin sind als Ursachen für Frakturen v. a. schwerere Traumatisierungen nach Stürzen im häuslichen Umfeld, Stürzen aus größerer Höhe, Verkehrsunfällen und auch der unkontrollierte Sturz aufgrund von Nebenerkrankungen, wie bei einer transitorisch ischämischen Attacke, bei einem entgleisten Diabetes mellitus oder Herzrhythmusstörungen, zu nennen.

\section{Frakturentstehung}

\section{Formen}

In Abhängigkeit vom Unfallmechanismus, von der Intensität der Gewalteinwirkung und der Knochenbeschaffenheit resultieren verschiedene Frakturformen. Hochrasanztraumen bei Motorrad- und Autounfällen weisen die höchste Morbidität bei distalen Femurfrakturen auf. Betroffen sind hauptsächlich junge Leute zwischen dem 20. und 30. Lebensjahr.

An 2. Stelle sind die Oberschenkelbrüche als Folge eines Sturzes in der Gruppe der 6o- bis 9o-Jährigen zu nennen. 
Der hohe Anteil älterer Frauen weist auf die Osteoporose als Hauptkausalfaktor hin [6]. Während beim osteoporotisch geschwächten Knochen des älteren Menschen bereits eine geringere Gewalteinwirkung ausreicht, bedarf es beim jungen Patienten erheblicher Kräfte, um das distale Femur brechen zu lassen [12].

\section{Gewalteinwirkung}

Suprakondyläre Femurfrakturen können durch direkte oder indirekte Gewalteinwirkung entstehen [25].

Direkte Gewalteinwirkung. Sie führt in Abhängigkeit von der Lokalisation der Krafteinwirkung zur Quer-, Trümmer-, mono- oder bikondylären Fraktur.

Beispiele für direkte Gewalteinwirkung sind die "Armaturenbrettverletzungen“ bei einem Aufprallunfall („dashbord injury“). Typisch bei Auffahrunfällen ist das Vorschieben der Beine unter das Armaturenbrett bei nicht angeschnalltem Fahrer/ Beifahrer. Durch das unmittelbar danach erfolgende Schleudern des Körpers nach vorn werden die Oberschenkel über die untere Armaturenbrettkante gehebelt. In Abhängigkeit der Lage der Kante in Bezug auf den Oberschenkel entstehen eine Femurschaft- oder eine suprakondyläre Femurfraktur. Beim Anprall des gebeugten Knies gegen einen festen Widerstand wird die Kniescheibe wie ein Keil zwischen die Kondylen getrieben und spaltet sie in der Ebene des kondylären Gleitlagers. Hierdurch werden die Kondylen voneinander getrennt und vom Schaft gesprengt. Zusätzlich - unter Umständen aber auch isoliert - bricht das Femur suprakondylär, wenn der distale Oberschenkel unter dem Lenkrad fixiert ist und der Körper nach vorne geworfen wird [25]. Die gleiche Gewalt kann über die Kraft übertragende Achse des Femurs ggf. zu einer Hüftgelenkluxation oder aber zu einer Luxationsfraktur führen.

Ein ähnlicher Unfallmechanismus liegt beim Sturz aus großer Höhe vor, wenn man in Hockstellung aufkommt. Je nach dem Ausmaß solcher Gewalt resultieren eine bikondyläre Längsfraktur oder bei geringer Gewalt ein Spaltungsbruch.

Ein anderer Frakturtyp, hervorgerufen durch direkte Gewalteinwirkung, ist die monokondyläre Fraktur des late-

Trauma Berufskrankh 2009 · 11 [Suppl 2]: 146-153 DOI 10.1007/s10039-008-1467-9

(c) Springer Medizin Verlag 2009

\section{S. Rein · R. Grass · H. Zwipp Suprakondyläre Femurfrakturen. Epidemiologie und Frakturklassifikation}

\section{Zusammenfassung}

Ziel der vorliegenden Arbeit war, die Epidemiologie und Frakturklassifikation von suprakondylären Femurfrakturen anhand der Literatur darzustellen. Die Häufigkeit dieser Verletzungen wird mit 4-31\% aller Femurfrakturen angegeben. Die Inzidenz beträgt $4,5 / 10^{5}$ Einwohner/Jahr bei einer Prävalenz von $0,4 \%$. Ursachen und Prädispositionen sind Hochrasanztraumen, Stürze aus großer Höhe, Implantation einer Knieendoprothese mit Stielkomponente und Vorhandensein einer femoralen Notch, Osteoporose, rheumatoide Arthritis und Erkrankungen, die mit einem erhöhten Sturzrisiko einhergehen. Die Altersverteilung ist sowohl bei Erwachsenen als auch bei Kindern zweigipflig. Prinzipiell werden direkte von indirekten Unfallmechanismen unterschieden. Die Dislokation rich- tet sich primär nach der Richtung der verursachenden Kraft und sekundär nach dem Zug der Muskulatur an den Frakturfragmenten. Begleitverletzungen umfassen Weichteilschäden, Bandverletzungen, Knorpelabscherfragmente und Meniskusschäden des Kniegelenkes. 1/3 der Patienten sind polytraumatisiert. Die AO-Klassifikation erlaubt eine sinnvolle Zuordnung aller Bruchformen und hat sich international durchgesetzt. Die epiphysären Frakturen werden nach Salter und Harris und/oder Aitken eingeteilt.

\section{Schlüsselwörter}

Suprakondyläre Femurfraktur .

Epidemiologie · Frakturklassifikation · Begleitverletzungen $\cdot$ Klassifikation

\section{Supracondylar fractures of the femur. Epidemiology and fracture classification}

\section{Abstract}

This paper reviews the epidemiology and fracture classification of supracondylar fractures of the femur. Supracondylar fractures comprise between $4 \%$ and $31 \%$ of all femoral fractures. The incidence is $4.5 / 10^{5}$ per year, with a prevalence of $0.4 \%$. Supracondylar fractures typically result from high-energy trauma, such as motor vehicle accidents, in younger patients and from low-energy injuries in the elderly. Consequently, the latter are frequently associated with osteoporosis, rheumatoid arthritis, knee endoprostheses, neurological disorders, and frequent falls. The amount of dislocation of the fracture is determined primarily by the acting force of the injury and secondarily by muscle pull. Additional injuries include soft tissue damage, meniscal and ligamentous lesions, and osteochondral fractures. One-third of all patients are polytraumatised. The fractures are classified using the $\mathrm{AO}$ classification. Physeal injuries in children and adolescents are differentiated using the classification of Salter and Harris and/or Aitken.

\section{Keywords}

Supracondylar femoral fractures . Epidemiology · Fracture classification . Additional injuries · Classification 


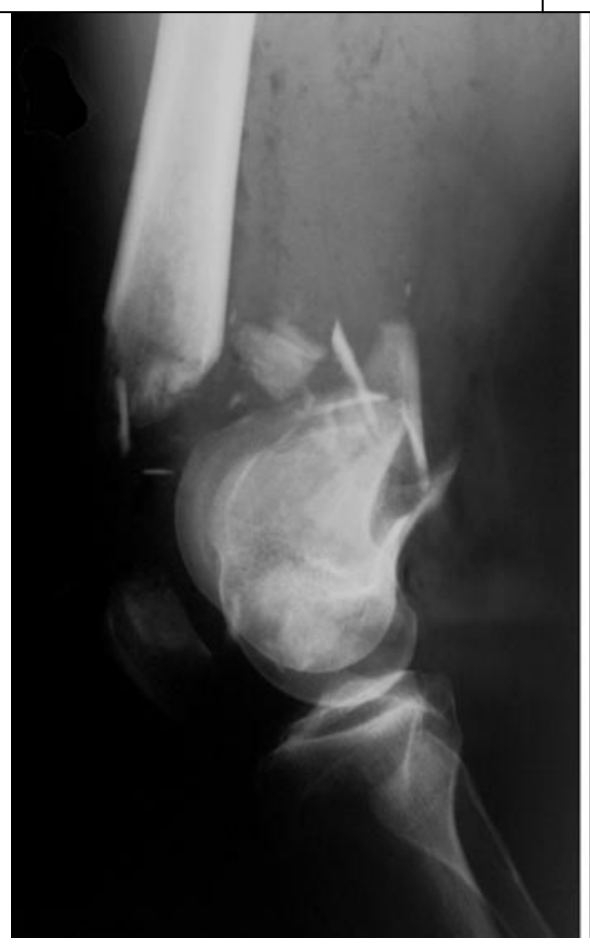

Abb. 1 \ Suprakondyläre Femurfraktur 33-A3 nach AO-Klassifikation, Ziehen des proximalen Anteils des distalen Fragments durch Zug des M. gastrocemius nach dorsal

ralen Femurkondylus bei Motorradfahrern. In sitzender Position und bei Beugung des Kniegelenks um etwa $90^{\circ}$ stellt der lateralen Femurkondylus den herausragenden Teil dar, wobei jedoch auch die Abduktion eine Rolle spielt.

Indirekte Gewalteinwirkung. Bei ihr wirkt die Kraft über die Längsachse des gesamten Beines [25]. Typisch sind Auffahrunfälle bei gestrecktem Kniegelenk, bei welchen das Tibiaplateau gegen die Kondylen getrieben wird. Da die Kompakta im unteren Femurdrittel mehr und mehr an Stärke abnimmt, kommt es in dieser unmittelbar suprakondylär gelegenen Zone zu einer entweder quer verlaufenden oder medial und lateral abgeschrägten Fraktur des Femurschaftes. Nachfolgend staucht sich der harte Femurschaft von oben in das Kondylenmassiv ein und kann seine Sprengung bewirken. Es entsteht ein bikondylärer Bruch [18]. Sind dagegen hierbei vorwiegend exzentrisch einseitige Kräfte im Spiel, führt diese Längsstauchung zu Abscherungen eines gesamten Kondylus oder - bei Beugestellung des Kniegelenks - der hinteren Anteile eines, meist des medialen, Kondylus (so genannte Hoffa-Fraktur) [25]. Es entsteht ein monokondylärer Bruch.
Viel seltener werden Spiralfrakturen in der suprakondylären Femurregion beobachtet. Sie entstehen bei den so genannten „twisting-injuries“, wenn der Körper sich um den festgestellten Unterschenkel dreht [22]. Ein solcher Unfallmechanismus liegt z. B. bei Skifahrern vor.

Wenn größere Gewalt einwirkt, ist auch der Weichteilmantel stärker in Mitleidenschaft gezogen. Dies spielt insbesondere nach Zweiradunfällen eine Rolle, bei denen häufig höhergradig offene Frakturen entstehen.

Die Dislokation bei den suprakondylären Frakturen wird primär durch die Richtung der verursachenden Kräfte während der initialen Frakturdislokation und sekundär durch den Zug der Beinmuskeln an den Frakturfragmenten hervorgerufen. Meist ziehen die Adduktoren das proximale Fragment nach medial. Durch den Zug des M. gastrocnemius wird das distale Fragment proximal nach dorsal gekippt, sodass gegenüber dem proximalen Fragment eine Achsenabweichung nach anterior resultiert (- Abb. 1). Diese Wirkung ist umso stärker, je näher die Fraktur zum Gelenk hin liegt.

Bei den dia- und transkondylären Frakturen kann die muskuläre Befestigung am jeweiligen Femurkondylus zum Abkippen und zur Rotationsfehlstellung führen.

\section{Begleitverletzungen}

Nur in $1 / 5$ der Fälle liegt die distale Femurfraktur als isolierte Verletzung vor, $1 / 3 \mathrm{der}$ jüngeren Patienten sind polytraumatisiert, die übrigen Betroffenen weisen Begleitverletzungen auf. Dabei ist die Anzahl offener (40\%) und geschlossener (etwa 35\%) Weichteilschäden als Begleitverletzungen der supra- und diakondylären Femurfrakturen hoch [25]. Bei fast der Hälfte der intraartikulären Brüche handelt es sich um offene Frakturen [15]. Der Anstieg in den letzten Jahren ist durch den höheren Anteil von Zweiradfahrern bedingt [25].

Neben der Traumatisierung des Weichteilmantels, der Kniegelenkbänder, Menisken und Gelenkknorpel der Kondylen sind Frakturen der Kniescheibe sowie Mittelfuß-, Fersenbein-, distale Unterschenkel-, Schenkelhals- oder Luxationsfrakturen und Abscherfrakturen des hinteren Pfannenrands des Azetabulums anzutreffen. Diese so genannten „Kettenverletzungen" entstehen durch die Längsstauchung des Beins und kommen bei etwa $20 \%$ der trans- und suprakondylären Frakturen vor.

Obwohl Nerven- (1\%) und Gefäßläsionen $(3 \%)$ als selten auftretende Begleitverletzungen gelten, sind traumatische Gefäßschädigungen bei den suprakondylären Brüchen öfter als bei allen anderen Femurfrakturlokalisationen anzutreffen [15]. So kann eine Luxation des Kniegelenks nach dorsal in $50 \%$ mit Gefäßverletzung der A. poplitea kombiniert sein.

Bandverletzungen sind als Begleitverletzungen bei knapp 10\% dieser Brüche zu finden [25]. Bei Kreuzbandverletzungen handelt es sich häufig um knöcherne proximale Ausrisse. Meniskusschäden finden sich bei bis zu 8\% der Verletzten. Hierbei handelt es sich in aller Regel um Ausrisse der in sich intakten Menisken aus ihrer Verankerung, nicht um schwerere intrameniskale Schäden.

Größere kartilaginäre und osteokartilaginäre Abscherfragmente, so genannte. "flakes", finden sich bei etwa 10\% dieser Brüche [25]. Der Unfallablauf macht verständlich, dass begleitende Patellabrüche relativ häufig - in bis zu $15 \%$ - angetroffen 
werden. Fast regelmäßig finden sich nach Anpralltraumen - auch ohne begleitende Patellafraktur - frische Schäden des retropatellaren Knorpels unterschiedlicher Schwere und Ausdehnung [25].

Ipsilaterale Frakturen von Femur und Tibia werden Floating-Knee-Verletzungen genannt und können aus Kombinationen von diaphysären, metaphysären und intraartikulären Frakturen bestehen [5]. Häufig sind sie durch Hochrasanztraumen bedingt, welche mit potenziell lebensbedrohlichen Verletzungen des Kopfes, des Thorax und des Abdomens im Sinne eines Polytraumas assoziiert sein können [3, 14]. Seitenband- und Meniskusverletzungen können ebenso mit diesem Frakturkomplex vergesellschaftet sein. Komplikationen, wie ein Kompartmentsyndrom oder initial nicht diagnostizierte Kniebandverletzungen und die Notwendigkeit einer Amputation im weiteren Verlauf, sind nicht selten. Adamson et al. [1] zeigten in ihrer Studie bei Typ-II-Frakturen eine Kniegelenkbeteiligung von 65\% der Femurfrakturen und $68 \%$ der tibialen Brüche. Bei 32\% der Patienten waren beide Frakturen intraartikulär [1]. Schwerwiegende Begleitverletzungen wiesen $71 \%$ der Patienten auf [1]. Offene Frakturen lagen in $62 \%$ der Fälle vor [1]. Begleitende vaskuläre Verletzungen waren in 21\% der Fälle vorhanden [1]. Die Floating-Knee-Frakturen sind außerdem häufig mit schweren Weichteilschäden kombiniert [3].

\section{Kinder}

Die distale Femurepiphyse trägt $\mathrm{zu} 70 \%$ des Längenwachstums des Femurs und $\mathrm{zu}$ 40\% des gesamten Wachstums der unteren Extremität bei [11]. Distale Femurfrakturen sind mit einer Häufigkeit von o,6-1,2\% aller Brüche im Kindes- und Jugendalter relativ selten $[17,19]$. Sie können als metaphysäre Stauchungsfrakturen, vollständige metaphysäre Frakturen, als Epiphysenlösungen und -frakturen sowie als Übergangsfrakturen vorkommen, wobei insbesondere Letztere selten sind. Gelegentlich treten sie als Kombinationsverletzung mit vollständiger oder teilweiser Epiphysenlösung plus zusätzlichem Ausbruch eines Anteils der Femurrolle in Erscheinung [19].

Tab. 1 Historische Klassifikationen der distalen Femurfrakturen. (Nach [27, 28, 29])

\begin{tabular}{|c|c|c|c|}
\hline Klassifikation & \multicolumn{2}{|l|}{ Typ } & Beschreibung \\
\hline \multirow{3}{*}{$\begin{array}{l}\text { Schatzker u. } \\
\text { Lambert [27], } \\
1979\end{array}$} & \multicolumn{2}{|l|}{ I } & Einfache Frakturen \\
\hline & \multicolumn{2}{|l|}{ II } & Mehrfragmentfrakturen ohne Gelenkbeteiligung \\
\hline & \multicolumn{2}{|l|}{ III } & Frakturen mit Gelenkbeteiligung \\
\hline \multirow{12}{*}{$\begin{array}{l}\text { Seinsheimer } \\
{[28], 1980}\end{array}$} & \multicolumn{2}{|l|}{ I } & Nicht disloziert \\
\hline & \multirow[t]{3}{*}{ II } & & Metaphysäre Fraktur ohne Beteiligung der interkondylären Notch \\
\hline & & a & Einfach \\
\hline & & b & Mehrfragmentär \\
\hline & \multirow[t]{4}{*}{ III } & & Frakturen mit Beteiligung der interkondylären Notch \\
\hline & & a & Fraktur des medialen Kondylus \\
\hline & & b & Fraktur des lateralen Kondylus \\
\hline & & c & Bikondyläre Fraktur \\
\hline & \multirow[t]{4}{*}{ IV } & & Intraartikuläre Femurkondylenfrakturen \\
\hline & & $\mathrm{a}$ & Medialer Kondylus einfach oder mehrfragmentiert \\
\hline & & $\mathrm{b}$ & Lateraler Kondylus einfach oder mehrfragmentiert \\
\hline & & c & Beide Kondylen und Notch mehrfragmentiert \\
\hline \multirow{5}{*}{$\begin{array}{l}\text { Shelbourne } \\
\text { u. Brückmann } \\
{[29], 1982}\end{array}$} & \multicolumn{2}{|l|}{1} & T- bzw. Y-förmige interkondyläre Fraktur \\
\hline & \multicolumn{2}{|l|}{ II } & Quere Fraktur \\
\hline & \multicolumn{2}{|l|}{ III } & Schräge Fraktur \\
\hline & \multicolumn{2}{|l|}{ IV } & Spiralfraktur \\
\hline & \multicolumn{2}{|l|}{ V } & Mehrfragmentfrakturen mit großen Knochenfragmenten \\
\hline
\end{tabular}

In einer Studie aus den USA wurden 1139 Kinder mit Femurfrakturen untersucht. Hierbei ergab sich ein Verteilungsmuster von $62,5 \%$ für Schaftfrakturen, gefolgt von $12,5 \%$ für proximale Frakturen und $11,7 \%$ für distale Femurfrakturen [24]. Der Altersverlauf war zweigipflig mit Häufigkeitsgipfeln unter dem 6. und nach dem 13. Lebensjahr [17].

Eid u. Hafez [11] ermittelten in ihrer retrospektiven Studie von 151 Kindern mit distaler Femurfraktur ein durchschnittliches Verletzungsalter von 12,3 Jahren bei einer Streuungsbreite von 10 Monaten-16 Jahre mit einer Verteilung für Jungen zu Mädchen von 6:1. In 60,3\% der Fälle war das linke, in $39,7 \%$ der Fälle das rechte $\mathrm{Fe}$ mur verletzt. Als Verletzungsursachen wurden sportliche Aktivitäten mit 59,6\%, Verkehrsunfälle mit 22,5\% und Stürze mit $17,9 \%$ angegeben. Offene Frakturen traten in 2,6\% der Fälle auf [11]. Bei Kindern bis zu 3 Jahren waren die Ursachen einer distalen Femurfraktur in $18,7 \%$ der Fälle nicht unfallbedingte Traumen (,,nonaccidental trauma"), in 16,7\% der Fälle kombinierte Schaft- und distale Femurfrakturen [24]. Als Begleitverletzungen wurden weitere knöcherne Verletzungen in 13,9\%, Peronäusparesen in $7,3 \%$, viszerale Verletzungen in $4,6 \%$ und Gefäßverletzungen in 2,6\% der Fälle beobachtet [11]. Nach der Klassifikation von Salter und Harris handelte es sich in der Studie von Eid u. Hafez [11] in 25,8\% der Fälle um Grad-I-, in $43 \%$ um Grad-II-, in $12,6 \%$ um GradIII-, in $14,6 \%$ um Grad-IV- und in $4 \%$ um Grad-V-Frakturen.

\section{Frakturklassifikationen}

Das Bestreben, durch Klassifizieren der distalen Femurfrakturen nach typischen Merkmalen Aussagen über Verletzungsschwere, Therapie und Prognose treffen zu können, führte zur Entwicklung verschiedener Schemata. Grundsätzlich wird in jeder Einteilung zwischen extraartikulärem, intraartikulärem und isoliertem kondylärem Frakturverlauf unterschieden. Als weitere Einteilungskriterien wurden Dislokationsgrad und -richtung, Vorhandensein von Trümmerzonen sowie Beteiligung der Gelenkfläche benutzt.

\section{Adulte Frakturen}

\section{Historische Klassifikationen}

Einteilung nach Schatzker u. Lambert. Nach dem Frakturverlauf werden 3 Typen unterschieden (• Tab. 1, [27]). 


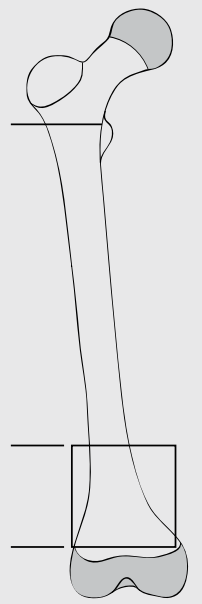

Abb. $2 \triangleleft$ AO-Klassifikation der Femurschaftfrakturen, Unterteilung des Femurs, Quadrat Bestimmung des metaphysären Anteils bei Kindern durch Anlegen eines Quadrats an der größten Kondylenbreite, weitere Erläuterung s. Text

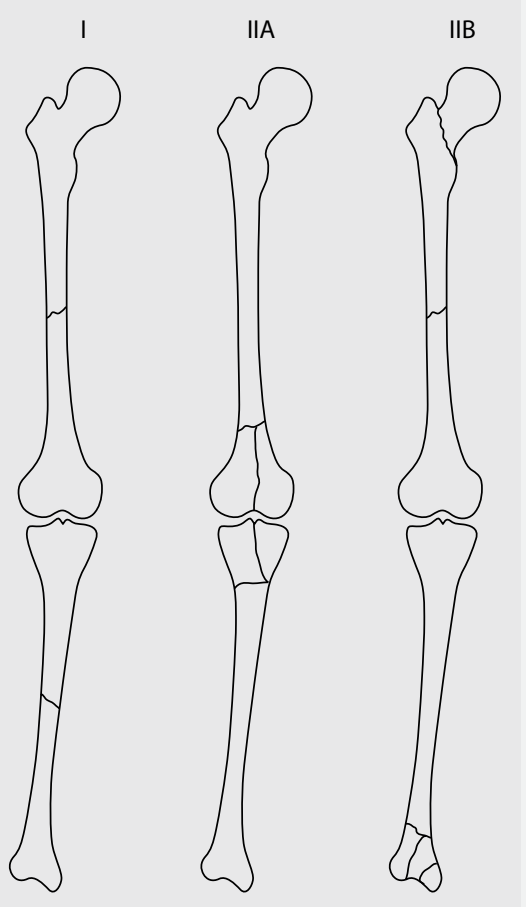

Seinsheimer-Klassifikation. Seinsheimer [28] berücksichtigte in seiner Einteilung den Frakturverlauf und das Ausmaß der knöchernen Zerstörung sowohl der Metaphyse als auch des jeweiligen Kondylus bei Gelenkbeteiligung. Die Frakturen werden in 4 Typen unterteilt (• Tab. 1, [28]).

\section{Einteilung nach Shelbourne u. Brück-} mann. 1982 kritisierten Shelbourne u. Brückmann [29] die mangelhafte therapeutische Hilfestellung vorhandener Klassifikationen supra- und transkondylärer Femurfrakturen und schlugen eine den
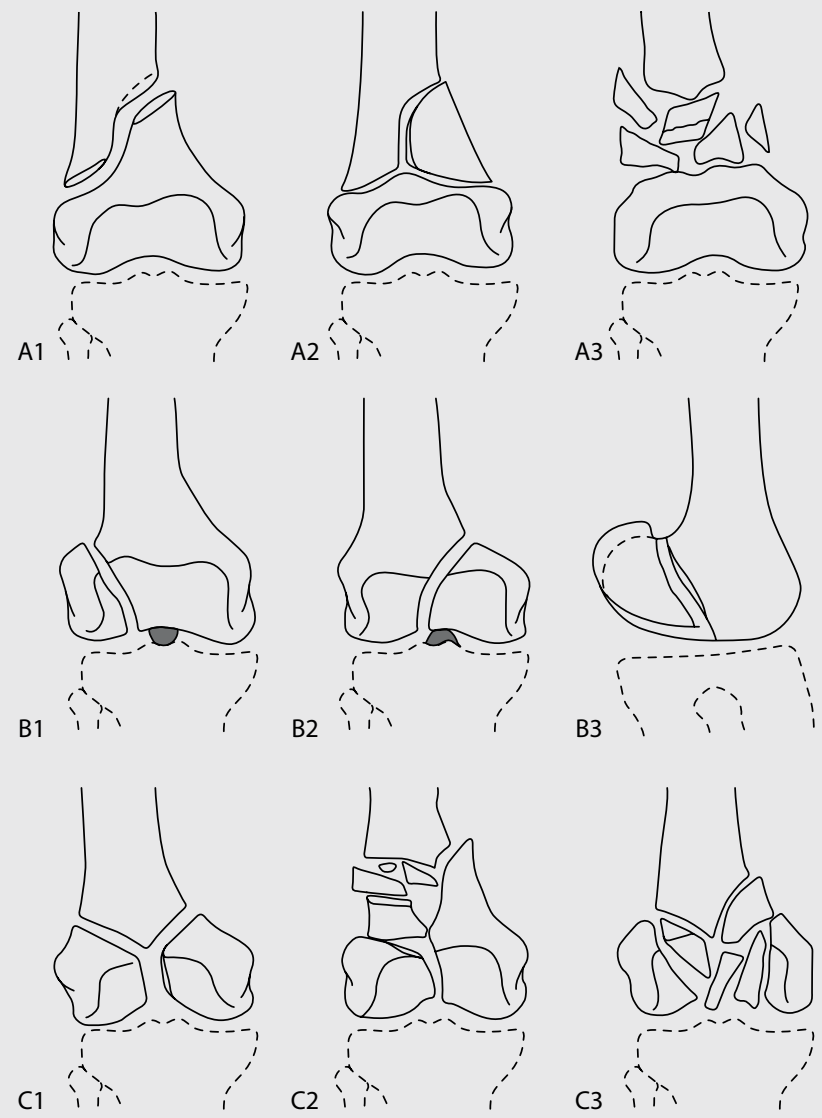

Abb. $3<$ AO-Klassifikation der suprakondylären Femurfrakturen, weitere Erläuterung s. Text

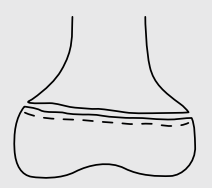

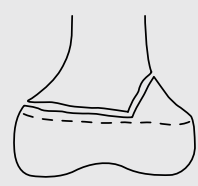

II

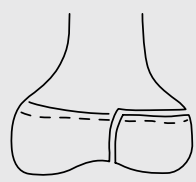

III

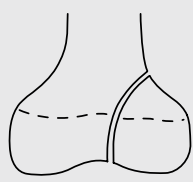

IV

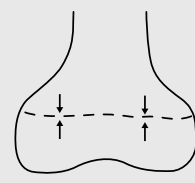

V

Abb. $5 \Delta$ Epiphysäre Fraktureinteilung in 5 Grade

Abb. $4 \varangle$ Schematische Darstellung der Floating-Knee-Klassifikation, weitere Erläuterung s. Text

Bruchverlauf beschreibende Einteilung vor (• Tab. 1, [29]).

\section{AO-Klassifikation}

Im deutschsprachigen Raum und international hat sich die von Müller et al. [20] 1987 empfohlene Klassifikation, die von der Arbeitsgemeinschaft für Osteosynthese (AO) im Grundsatz übernommen wurde, durchgesetzt. In dieser werden die distalen Femurfrakturen nach anatomischen Gesichtspunkten aufgrund der Frakturlokalisation eingeteilt als

- distale Femurschaftfrakturen (bis zum Beginn der trompetenförmigen
Femurerweiterung und Kortikalisverjüngung),

- suprakondyläre Femurfrakturen (von der trompetenförmigen Femurerweiterung und Kortikalisverjüngung bis zur Ansatzstelle der Seitenbänder, dem distalen Femurabschnitt in dem Quadrat über der größten Kondylenbreite im a.-p. Röntgenbild entsprechend),

- trans- oder diakondyläre Femurfrakturen oder auch als

Kombination der genannten Frakturtypen. 


\begin{tabular}{|c|c|c|c|c|c|c|c|c|c|c|c|}
\hline \multicolumn{5}{|l|}{ Lokalisation } & \multicolumn{7}{|c|}{ Morphologie } \\
\hline \multirow[t]{2}{*}{ Knochen } & \multirow{2}{*}{\multicolumn{2}{|c|}{ Segment }} & \multirow{2}{*}{\multicolumn{2}{|c|}{ Subsegment }} & \multicolumn{4}{|c|}{ Kind } & \multirow{2}{*}{\multicolumn{2}{|c|}{ Schwere }} & \multirow[t]{2}{*}{ Dislokation } \\
\hline & & & & & \multicolumn{2}{|c|}{ Epiphyse } & \multicolumn{2}{|c|}{ Metaphyse } & & & \\
\hline 1 Humerus & 1 & Proximal & $\mathrm{D}$ & Diaphyse & $\mathrm{E} / 1$ & Salter-Harris I & & & 1 & Einfach & \multirow{9}{*}{$\begin{array}{l}\text { Keine Klas- } \\
\text { sifikation für } \\
\text { suprakondyläre } \\
\text { Femurfrakturen }\end{array}$} \\
\hline 2 Radius/Ulna & 2 & Schaft & M & Metaphyse & $E / 2$ & Salter-Harris II & $M / 2$ & $\begin{array}{l}\text { Wulstbruch } \\
\text { Grünholzfraktur }\end{array}$ & 2 & Komplex & \\
\hline 3 Femur & 3 & Distal & $E$ & Epiphyse & $E / 3$ & Salter-Harris III & $M / 3$ & komplette Fraktur & & & \\
\hline \multirow[t]{6}{*}{4 Tibia/Fibula } & & & & & $E / 4$ & Salter-Harris IV & & & & & \\
\hline & & & & & $\mathrm{E} / 5$ & Tillaux-Fraktur (2 Ebenen) & & & & & \\
\hline & & & & & $\mathrm{E} / 6$ & Tillaux-Fraktur (3 Ebenen) & & & & & \\
\hline & & & & & $\mathrm{E} / 7$ & Ligamentavulsion & $\mathrm{M} / 7$ & Ligamentavulsion & & & \\
\hline & & & & & $\mathrm{E} / 8$ & Abscherfraktur & & & & & \\
\hline & & & & & $E / 9$ & Andere & $M / 9$ & Andere & & & \\
\hline
\end{tabular}

Dabei wird das Femur mit der Zahl 3 verschlüsselt, und nachfolgend werden der proximale Anteil mit 1, der diaphysäre Anteil mit 2 und der distale Anteil mit 3 beziffert. Bei kindlichen Frakturen wird Letzterer in einen meta- und einen epiphysären Anteil unterschieden, welcher durch die Wachstumsfuge unterteilt wird. Die Größe des metaphysären Anteils ergibt sich aus einem Quadrat, welches an der größten Kondylenbreite im a.-p. Röntgenbild angelegt wird (• Abb. 2).

Die Vorteile dieser Klassifikation liegen einerseits in dem einfachen und pragmatischen Aufbau, welcher durch umfangreiche Subgruppenunterteilung eine sehr detaillierte Frakturaufschlüsselung erlaubt, und andererseits in der Möglichkeit, anhand des ermittelten Frakturmusters bereits Vorstellungen über das in Frage kommende osteosynthetische Verfahren und die allgemeine Heilungsprognose zu entwickeln.

Den Frakturverlauf berücksichtigend sind für das distale Femur 3 Hauptgruppen zu unterscheiden. Diese werden weiter differenziert, bei den extraartikulären Frakturen (Typ A) in

- einfache Frakturen (A1),

- Frakturen mit metaphysärem Keil (A2) und

- die mehrfach metaphysäre Fraktur (A3),

bei den partiell artikulären Frakturen (Typ B) in

- sagittale Frakturen des Condylus lateralis $(\mathrm{B} 1)$,
- sagittale Frakturen des Condylus medialis $\left(\mathrm{B}_{2}\right)$ und

frontale Frakturen (B3)

und bei den vollständig artikulären Typ-

C-Frakturen in

- artikulär und metaphysär einfache (C1),

- artikulär einfache und metaphysär mehrfache $\left(\mathrm{C}_{2}\right)$ und

artikulär mehrfache Frakturen $\left(\mathrm{C}_{3}\right)$

- Abb. 3, [20]). Eine weitere Unterteilung in Subgruppen ermöglicht die genauere Aufschlüsselung in Bezug auf den Umfang der knöchernen Beteiligung und den Frakturverlauf [20].

\section{Floating-Knee-Klassifikation}

1975 prägten Blake u. McBryde [5] den Begriff „floating knee“, welcher frei mit „tanzendem Knie" übersetzt werden kann und kombinierte ipsilaterale Frakturen des Femurs und der Tibia beschreibt. Der Begriff „wahre“ („true“) oder auch Typ-IFraktur wird für reine diaphysäre Frakturen ohne intraartikuläre Kniegelenkbeteiligung, die Bezeichnung „variable“ („variant“) bzw. Typ-II-Fraktur wird für die Involvierung von einem oder mehreren Gelenken verwendet [5]. Hierbei sind bei Typ IIA das Kniegelenk allein, bei Typ IIB das Hüft- oder Sprunggelenk ebenfalls betroffen (• Abb. 4, [5]).

\section{Kindliche Frakturen}

Im Gegensatz zu den adulten Frakturen besteht die primäre Schwierigkeit der kindlichen Brüche nicht in ihrer Komplexizität oder Schwere, sondern in ihrem Phänomen des Wachstums. Diese Mechanismen sind noch nicht vollständig verstanden, aber sie können den Heilungsprozess in Abhängigkeit von Frakturstruktur und vom Alter des Patienten beeinflussen [31].

\section{Historische Klassifikationen}

Die von Salter u. Harris [26] 1963 entwickelte Klassifikation teilt die epiphysären Frakturen in 5 Grade ein (• Abb. 5). Dabei entsprechen:

- Grad I einer Epiphyseolyse ohne Begleitfraktur,

- Grad II einer partiellen Epiphyseolyse mit metaphysärer Begleitfraktur,

- Grad III einer partiellen Epiphyseolyse mit epiphysären Begleitfraktur,

- Grad IV einer epimetaphysären Fraktur und

- Grad V einer unvollständigen oder vollständigen Kompression der Epiphyse, einer so genannten „CrushVerletzung“.

Die von Aitken [2] vorgenommene Klassifikation der epiphysären Frakturen beinhaltet:

- bei Typ I eine partielle Epiphyseolyse mit metaphysärer Begleitfraktur (entspricht Grad II nach Salter u. Harris),

- bei Typ II eine partielle Epiphyseolyse mit epiphysärer Begleitfraktur (entspricht Grad III nach Salter u. Harris) und 
Knieverletzungen

\begin{tabular}{|c|c|c|c|c|c|}
\hline \multicolumn{3}{|c|}{ Einfache Frakturen } & \multicolumn{3}{|c|}{ Keil-/ Multifragmentäre Frakturen } \\
\hline Kode & Abbildung & Beschreibung & Kode & Abbildung & Beschreibung \\
\hline $33-M / 2.1$ & & Wulstfraktur & & & \\
\hline $33-M / 3.1$ & & Einfach komplett & $33-M / 3.2$ & & Multifragmentär \\
\hline 33-M/7.1 & & $\begin{array}{l}\text { Ligamentavulsion } \\
\text { bilateral }\end{array}$ & & & \\
\hline $33 \mathrm{tM} / 7.1$ & & $\mathrm{t}=$ tibial $/$ medial & & & \\
\hline 33f-M/7.1 & & $f=$ fibular/lateral & & & \\
\hline
\end{tabular}

Abb. $6 \Delta$ AO-Klassifikation der distalen metaphysären kindlichen Femurfrakturen, 33-M

\begin{tabular}{|c|c|c|c|c|c|}
\hline \multicolumn{3}{|c|}{ Einfache Frakturen } & \multicolumn{3}{|c|}{ Keil-/ Multifragmentäre Frakturen } \\
\hline Kode & Abbildung & Beschreibung & Kode & Abbildung & Beschreibung \\
\hline $33-E / 1.1$ & & $\begin{array}{l}\text { Einfache } \\
\text { Epiphyseolyse }\end{array}$ & & & \\
\hline $33-E / 2.1$ & & $\begin{array}{l}\text { Einfache } \\
\text { Epiphyseolyse mit } \\
\text { metaphysärem } \\
\text { Keil }\end{array}$ & $33-E / 2.2$ & & $\begin{array}{l}\text { Epiphyseolyse mit } \\
\text { multifragmentärem } \\
\text { metaphysärem } \\
\text { Keil }\end{array}$ \\
\hline $33-E / 3.1$ & & $\begin{array}{l}\text { Einfache } \\
\text { epiphysäre } \\
\text { Fraktur }\end{array}$ & $33-E / 3.2$ & & $\begin{array}{l}\text { Multifragmentäre } \\
\text { epiphysäre } \\
\text { Fraktur }\end{array}$ \\
\hline $33-E / 4.1$ & & $\begin{array}{l}\text { Einfache } \\
\text { epimetaphysäre } \\
\text { Fraktur }\end{array}$ & $33-E / 4.2$ & & $\begin{array}{l}\text { Multifragmentäre } \\
\text { epimetaphysäre } \\
\text { Fraktur }\end{array}$ \\
\hline $33-E / 8.1$ & & $\begin{array}{l}\text { Einzelne } \\
\text { intraartikuläre } \\
\text { Abscherfraktur }\end{array}$ & $33-E / 8.2$ & & $\begin{array}{l}\text { Multiple } \\
\text { intraartikuläre } \\
\text { Abscherfraktur }\end{array}$ \\
\hline
\end{tabular}

Abb. 7 \ AO-Klassifikation der distalen epiphysären kindlichen Femurfrakturen, 33-E 
teilung von Salter u. Harris und/oder Aitken differenziert.

\section{Korrespondenzadresse}

\section{Dr. S. Rein}

Klinik und Poliklinik für Unfallund Wiederherstellungschirurgie, Universitätsklinikum Carl Gustav Carus, Fetscherstraße 74, 01307 Dresden susanne.rein@uniklinikum-dresden.de

Interessenkonflikt. Der korrespondierende Autor gibt an, dass kein Interessenkonflikt besteht.

\section{Literatur}

1. Adamson GJ, Wiss DA, Lowery GL, Peters CL (1992) Type II floating knee: ipsilateral femoral and tibial fractures with intraarticular extension into the knee joint. J Orthop Trauma 6:333-339

2. Aitken AP (1965) Fractures of the epiphyses. Clin Orthop 41:19-23

3. Anastopoulos G, Assimakopoulos A, Exarchou E, Pantazopoulos T (1992) Ipsilateral fractures of the femur and tibia. Injury 23:439-441

4. Arneson TJ, Melton LJ 3rd, Lewallen DG, O'Fallon WM (1988) Epidemiology of diaphyseal and distal femoral fractures in Rochester, Minnesota, 19651984. Clin Orthop 234:188-194

5. Blake R, McBryde A Jr (1975) The floating knee: ipsilateral fractures of the tibia and femur. South Med J 68:13-16

6. Blatter G, Konig H, Janssen M, Magerl F (1994) Primary femoral shortening osteosynthesis in the management of comminuted supracondylar femoral fractures. Arch Orthop Trauma Surg 113:134-137

7. Court-Brown CM, Caesar B (2006) Epidemiology of adult fractures: a review. Injury 37:691-697

8. Culp RW, Schmidt RG, Hanks G et al (1987) Supracondylar fracture of the femur following prosthetic knee arthroplasty. Clin Orthop 222:212-222

9. Cusick RP, Lucas GL, McQueen DA, Graber CD (2000) Construct stiffness of different fixation methods for supracondylar femoral fractures above total knee prostheses. Am J Orthop 29:695-699

10. Ecke H, Neubert C, Neeb W (1980) Analyse der Behandlungsergebnisse von 1127 Patienten mit Oberschenkelfrakturen aus der Bundesrepublik Deutschland und der Schweiz. Unfallchirurg 6:3843

11. Eid AM, Hafez MA (2002) Traumatic injuries of the distal femoral physis. Retrospective study on 151 cases. Injury 33:251-255

12. El-Kawy S, Ansara S, Moftah A et al (2007) Retrograde femoral nailing in elderly patients with supracondylar fracture femur; is it the answer for a clinical problem? Int Orthop 31:83-86

13. Hernigou $P$, Mathieu G, Filippini $P$, Demoura A (2006) Facteurs du risque de fracture du fémur distal dans les prothèses totales du genou: Étude de 32 fractures per et postopératoires. Rev Chir Orthop Reparatrice Appar Mot 92:140-147

14. Hung SH, Lu YM, Huang HT et al (2007) Surgical treatment of type II floating knee: comparisons of the results of type IIA and type IIB floating knee. Knee Surg Sports Traumatol Arthrosc 15:578-586

15. Kinzl L (2000) Femur: distal. In: Rüedi TP, Buckley RE, Moran CG (eds) AO Principles of fracture management. Thieme, Stuttgart, pp 469-480
16. Kolmert L, Wulff K (1982) Epidemiology and treatment of distal femoral fractures in adults. Acta Orthop Scand 53:957-962

17. Kraus R, Schneidmüller D, Röder C (2005) Häufigkeit von Frakturen der langen Röhrenknochen im Wachstumsalter. Dtsch Ärztebl 102:838-842

18. Kuner EH (1975) Ursachen, Formen und Begleitverletzungen der distalen Oberschenkelfraktur. Hefte Unfallheilkd 120:1-8

19. Laer L von (2007) Das verletzte Kind. Thieme, Stuttgart

20. Müller ME, Nazarian S, Koch P (1987) Classification $A O$ des fractures: les os longs. Springer, Heidelberg

21. Nast-Kolb D, Kessler S, Duswald KH et al (1986) Extremitätenverletzungen polytraumatisierter Patienten: stufengerechte Behandlung. Unfallchirurg 89:149-154

22. Neer CS 2nd, Grantham SA, Shelton ML (1967) Supracondylar fracture of the adult femur. A study of one hundred and ten cases. J Bone Joint Surg Am 49:591-613

23. Regazzoni P, Leutenegger $A$, Ruedi T, Staehelin $F$ (1986) Erste Erfahrungen mit der dynamischen Kondylenschraube (DCS) bei distalen Femurfrakturen. Helv Chir Acta 53:61-64

24. Rewers A, Hedegaard H, Lezotte D et al (2005) Childhood femur fractures, associated injuries and sociodemographic risk factors: a population-based study. Pediatrics 115:e543-e552

25. Rüter A, Trentz O, Wagner M (2004) Distales Femur. In: Rüter A, Trentz O, Wagner M (Hrsg) Unfallchirurgie. Urban \& Fischer, München, S 1013-1028

26. Salter RB, Harris WR (1963) Injuries involving the epiphyseal plate. J Bone Joint Surg Am 45:587622

27. Schatzker J, Lambert DC (1979) Supracondylar fractures of the femur. Clin Orthop 138:77-83

28. Seinsheimer F 3rd (1980) Fractures of the distal femur. Clin Orthop 153:169-179

29. Shelbourne KD, Brückmann FR (1982) Rush-pin fixation of supracondylar and intercondylar fractures of the femur. J Bone Joint Surg Am 64:161169

30. Sisto DJ, Lachiewicz PF, Insall JN (1985) Treatment of supracondylar fractures following prosthetic arthroplasty of the knee. Clin Orthop 196:265-272

31. Slongo T, Audige L, Clavert JM et al (2007) The AO comprehensive classification of pediatric long-bone fractures: a web-based multicenter agreement study. J Pediatr Orthop 27:171-180

32. Su ET, Kubiak EN, Dewal H et al (2006) A proposed classification of supracondylar femur fractures above total knee arthroplasties. J Arthroplasty 21:405-408 